\title{
BMJ Open Protocol for a multicentre longitudinal mixed-methods study: feeding and survivorship outcomes in previously healthy young paediatric Intensive care survivors (the PIES Study)
}

\author{
Kathryn Morton (D) ,1,2 Anne-Sophie Emma Darlington, ${ }^{2}$ L V Marino ${ }^{3,4}$
}

To cite: Morton K, Darlington ASE, Marino LV. Protocol for a multicentre longitudinal mixed-methods study: feeding and survivorship outcomes in previously healthy young paediatric Intensive care survivors (the PIES Study). BMJ Open 2020;10:e041234. doi:10.1136/ bmjopen-2020-041234

- Prepublication history for this paper is available online. To view these files, please visit the journal online (http://dx.doi. org/10.1136/bmjopen-2020041234).

Received 03 June 2020 Revised 22 0ctober 2020 Accepted 16 November 2020

Check for updates

(C) Author(s) (or their employer(s)) 2020. Re-use permitted under CC BY-NC. No commercial re-use. See rights and permissions. Published by BMJ.

For numbered affiliations see end of article.

Correspondence to Mrs Kathryn Morton; kathryn.morton@uhs.nhs.uk

\section{ABSTRACT}

Introduction An admission to paediatric intensive care unit (PICU) is associated with multiple physical and environmental stressors, often involving many negative and painful oral experiences. Evidence from children with complex medical conditions suggests that feeding difficulties post-PICU stay are common, causing significant parental anxiety. Adult intensive care unit (ICU) survivor studies suggest feeding issues lasting up to 3 months post-discharge from ICU. There is, however, a paucity of evidence regarding feeding outcomes for previously healthy children following a PICU admission and whether painful oral experiences during an admission contribute to feeding difficulties post-discharge, negatively impacting on parental/caregiver anxiety.

Methods and analysis This longitudinal mixed-methods study will explore the impact of feeding difficulties, identifying any clinical risk factors during the first 6 months of PICU discharge in previously healthy young children ( $\leq 4$ years). Parents/caregivers of children will be asked to complete questionnaires relating to: feeding difficulties, parental/caregiver stress, and child and parental/caregivers' feeding behaviours at the point of PICU discharge, 1, 3 and 6 months post-discharge. Parents/caregivers will be invited to participate in qualitative semistructured interviews at 3 and 6 months post-PICU discharge exploring parental/caregiver experiences of feeding their child after PICU. Statistical analysis of the survey data will consist of descriptive and inferential statistics, plus qualitative analysis of any free text comments using thematic analysis.

Ethics and dissemination This study will provide an insight and increase our understanding of the prevalence of feeding difficulties in previously healthy children admitted to PICU and parental/caregiver experiences. Multiple methods will be used to ensure that the findings are effectively disseminated to service users, clinicians, policy and academic audiences. The study has full ethical approval from the National Health Service Research Ethics Committee (Ref: 20/YH/0160) and full governance clearance.

\section{INTRODUCTION}

Paediatric intensive care unit (PICU) admissions have increased by $15 \%$ over the last
Strengths and limitations of this study

- A strength of this study is that it is the first multicentre, longitudinal study to investigate feeding and survivorship outcomes in young paediatric intensive care unit (PICU) survivors, in the first 6 months post-discharge.

- By using a mixed-methods design, this study will provide a greater breadth of understanding into the prevalence and impact of feeding issues in previously healthy young children who survive PICU.

- A strength of this study's qualitative data collection method (interviews with parents/caregivers) lies in its ability to generate a rich narrative data set exploring the survivorship journey of families post-PICU.

- The longitudinal study design will allow us to explore any feeding difficulties over a 6-month period postPICU, potentially identifying transient and persistent problems.

- A limitation to the study's longitudinal design lies in its potential for high attrition which may affect data at 6 months, challenging the internal validity of the reported results.

decade. ${ }^{12}$ Approximately $70 \%$ are admitted due to emergency unplanned admissions, ${ }^{2}$ causing a period of distress and crisis for families. ${ }^{3}$ In developed countries, advances in medical care and technology mean that over $96 \%$ of PICU patients are discharged alive, ${ }^{4}$ although morbidity among childhood survivors is high. ${ }^{5}$ As a result, the focus of critical care has moved to improving survivorship, aiming to optimise physical, social, emotional, cognitive and functional outcomes for children and their families. ${ }^{6}$

Until now, there has been little focus on the impact an admission to PICU may have on oral feeding ability in survivors of critical illness. During an admission to PICU, children are exposed to multiple physical and environmental stressors, involving up to 
89 painful oral experiences, including the use of endotracheal tubes (ETT), extubations and re-intubations, nasogastric tube (NGT) insertion and frequent oral suctioning. ${ }^{7}$ These traumatic and often painful oral experiences have been linked to swallowing and eating difficulties in adult survivors of intensive care, ${ }^{8-12}$ with difficulties in self-feeding, reduced appetite, altered taste and food preferences lasting up to 3 months post-intensive care unit discharge. ${ }^{12} 13$

Despite most PICUs in the UK incorporating early nutritional support within 24 hours of admission, ${ }^{14} 15$ it is usual for children not to eat or drink orally during their intensive care admission. ${ }^{16}$ NGT feeding is routinely used during critical illness as a primary method of delivering nutrition support, ${ }^{16}$ resulting in young children missing out on normal oral feeding experiences. ${ }^{17} 18$ The impact of prolonged NGT feeding is well described, with evidence indicating that children under 1 year of age can take up to 2 years to establish oral feeding if they are NGT fed for significant periods of time. ${ }^{1920}$

Feeding is a complex learnt behaviour, occurring during infancy involving developmental maturation to coordinate the process of sucking, swallowing and breathing. This then advances into chewing and texture control..$^{21}$ There is also a social aspect of feeding, involving parental-child interactions, ${ }^{22}$ with parental behaviours and feeding styles directly influencing feeding behaviours of young children. ${ }^{20}{ }^{23}$ Parental feeding styles have been shown to influence food enjoyment, fussiness, food responsiveness, food neophobia and self-regulation in children. ${ }^{22}$ Parental feeding interactions and practices during childhood cancer treatment, for example, include pressurising children to eat, using food as rewards and bribes and being overindulgent, with the stress of eating having a negative effect on the parental-child relationship. ${ }^{324}$ There is, however, no evidence looking at feeding difficulties and parental-child feeding interactions associated with feeding in the previously healthy PICU population. ${ }^{25}$ Although there is some information describing feeding outcomes in children born prematurely and young children with coronary heart disease (CHD), there remains a lack of high-quality evidence. The consequence of an admission to PICU on the ability of young children to eat and drink initially after PICU discharge and then once home, and the implications this has for young children and their families, is not known.

\section{METHODS AND ANALYSIS \\ Study aims}

The PIES Study (feeding and survivorship outcomes in previously healthy young Paediatric IntensivE care $\underline{\text { Survi- }}$ vors) has six specific objectives:

1. To characterise and measure the prevalence of feeding difficulties in previously healthy children ( $\leq 4$ years) who survive critical illness during the first 6 months after PICU discharge.
2. To identify clinical predictors for the development of feeding difficulties in previously healthy young children ( $\leq 4$ years) who survive critical illness.

3 . To identify parental/caregiver feeding styles for previously healthy young children ( $\leq 4$ years) who survive critical illness.

4. To measure parental stress in parents/caregivers of previously healthy young children ( $\leq 4$ years) who survive critical illness.

5. To identify behaviours of previously healthy young children ( $\leq 4$ years) who survive critical illness.

6 . To develop an in-depth understanding of how parents/caregivers of previously healthy young children ( $\leq 4$ years old) who survive critical illness construct, experience and make sense of their survivorship journey from PICU admission, specifically looking at feeding experiences and parental-child relationships.

\section{Study design}

Based on the research question and objectives, a prospective, longitudinal mixed-methods design will be used. Quantitative and qualitative data will be collected simultaneously over several times points, analysed separately and then integrated giving equal emphasis to each strand. ${ }^{26}$ Parents/caregivers of children will be asked to take part in a longitudinal survey, completing questionnaires considering aspects relating to: feeding difficulties, parental/ caregiver stress, and child and parental/caregivers' feeding behaviours at the point of PICU discharge and at 1, 3 and 6 months post-discharge. Parents/caregivers will also be invited to participate in qualitative semistructured interviews at 3 and 6 months post-PICU discharge, which will explore parental/caregiver experiences of feeding their child post-PICU. Routinely collected clinical data about the PICU admission will additionally be collected. See figure 1 for schematic overview of the study design.

\section{Setting}

Participants will be recruited from up to 10 PICUs across the UK chosen to include variation in unit size, case mix, geographical location and patient demographic.

\section{Sample and recruitment}

\section{Eligibility criteria}

The chosen inclusion criteria will allow recruitment of previously healthy young children ( $\leq 4$ years) who are admitted to PICU both electively and in emergency situations. Participants will be eligible if they are parents/ caregivers (aged $\geq 18$ years of age) of a previously healthy child aged $\leq 4$ years who has received invasive ventilation for 48 hours or more (including at referring hospital if applicable) (table 1).

A limit of $\leq 4$ years of age has been set because the majority of children admitted to PICU are under school age, with children under 5 years of age spending the most number of days in PICU. ${ }^{4}$ Furthermore, the skills and behaviours learnt in the first few years of life are seen as imperative for future eating skills, attitudes and 


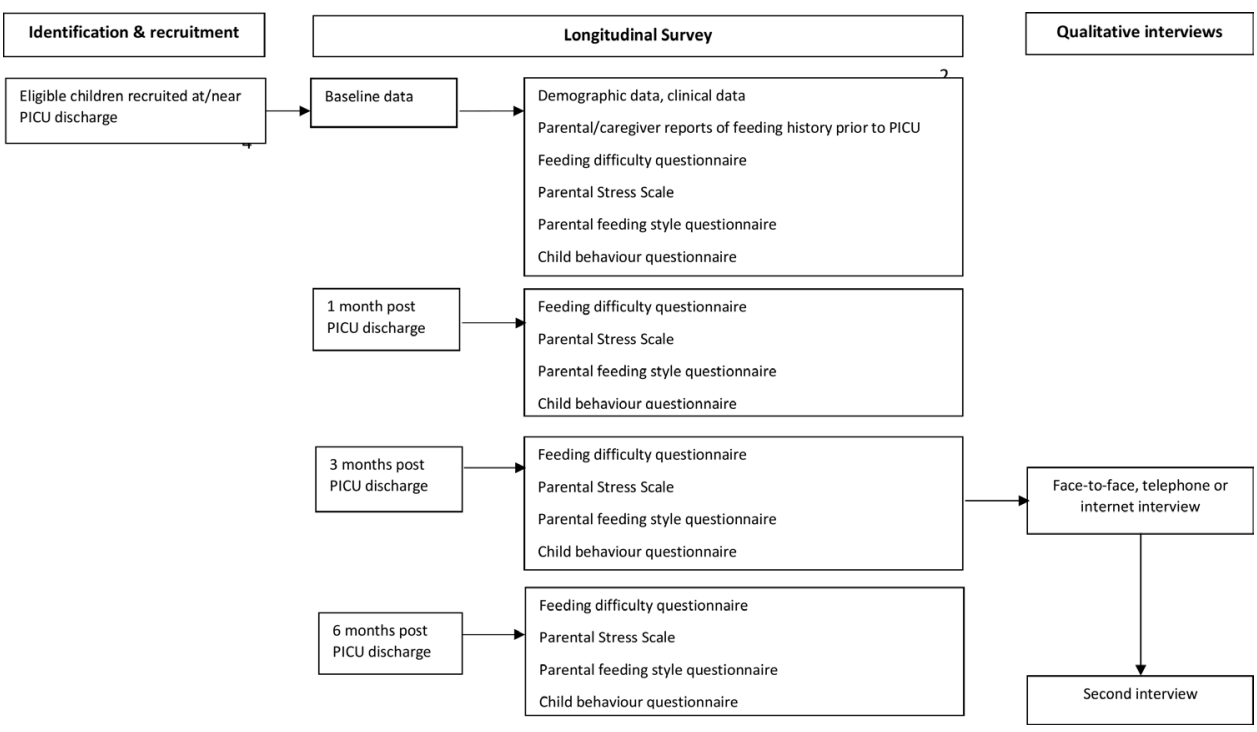

Figure 1 For schematic overview of the study design. PICU, paediatric intensive care unit.

behaviours needed for future adult health. ${ }^{27}$ Additionally, by studying this age range, any feeding difficulties that may occur during critical time-sensitive developmental feeding milestone windows, may also be identified. ${ }^{28}$ These include:

\section{Table 1 Eligibility criteria}

\begin{tabular}{|c|c|}
\hline Inclusion criteria & Rationale \\
\hline $\begin{array}{l}\text { Parents/caregivers (aged } \geq 18 \\
\text { years of age) of previously } \\
\text { healthy children aged } \leq 4 \text { years } \\
\text { who are ready to be discharged } \\
\text { from PICU }\end{array}$ & $\begin{array}{l}\text { Age limit required to } \\
\text { comply with the Research } \\
\text { Governance Framework for } \\
\text { Health and Social Care }{ }^{52}\end{array}$ \\
\hline $\begin{array}{l}\text { Parents/caregivers who have } \\
\text { sufficient language skills to } \\
\text { read the Participant Information } \\
\text { Sheet and to complete the } \\
\text { questionnaires in English }\end{array}$ & $\begin{array}{l}\text { Unable to translate study } \\
\text { materials into different } \\
\text { languages due to limited } \\
\text { study resources }\end{array}$ \\
\hline $\begin{array}{l}\text { Children are included if they: } \\
\text { are } \leq 4 \text { years } \\
\text { have received invasive } \\
\text { ventilation for } 48 \text { hours or } \\
\text { more (including at referring } \\
\text { hospital if applicable) }\end{array}$ & $\begin{array}{l}\text { To cover children up to } \\
\text { school age } \\
\text { Used as an indicator of } \\
\text { critical illness and seen } \\
\text { in adult ICU survivors to } \\
\text { affect swallowing and } \\
\text { feeding problems }\end{array}$ \\
\hline Exclusion criteria & Rationale \\
\hline Aged $>5$ years or older & $\begin{array}{l}\text { Age beyond preschool } \\
\text { years }\end{array}$ \\
\hline $\begin{array}{l}\text { Children not invasively } \\
\text { ventilated (so no ETT) }\end{array}$ & $\begin{array}{l}\text { Unable to fulfil inclusion } \\
\text { criteria }\end{array}$ \\
\hline $\begin{array}{l}\text { Children with previous feeding } \\
\text { difficulties (children who were } \\
\text { not fully orally fed prior to PICU } \\
\text { admission or have document } \\
\text { oral feeding difficulties) }\end{array}$ & $\begin{array}{l}\text { Unable to fulfil inclusion } \\
\text { criteria and unable to } \\
\text { consume sufficient } \\
\text { nutrients orally }\end{array}$ \\
\hline
\end{tabular}

ETT, endotracheal tubes; ICU, intensive care unit; PICU, paediatric intensive care unit.
- The initial feeding skill that is required to successfully breast or bottle feed at birth.

- To identify feeding difficulties that might occur during the weaning to complementary food stage (4-6 months of age), for example involving problems with textures, tastes and chewing.

- To identify feeding difficulties that might occur during the transition to autonomous child self-feeding during preschool years.

- To identify extreme cases of behaviour often associated with picky or fussy behaviour in preschool-aged children.

- Once children start school (>4 years of age), parents often have less control over lunchtime behaviours and food intake. ${ }^{29}$

The exclusion of non-English-speaking families is a limitation of the study design in terms of selection bias and may affect the generalisability of the results. This will be investigated in the interpretations of the study results and implications for clinical practice.

\section{Sample size}

Quantitative study

The sample size is based on estimating prevalence to a certain level of precision as defined by a $95 \%$ CI. Assuming a potentially low prevalence of just $20 \%$ (which is less than the neonatal intensive care unit and CHD population owing to their underlying baseline disease ${ }^{30-34}$ ), a sample size of 204 child participants would be sufficient to estimate feeding difficulty prevalence. Anticipating a $40 \%$ drop-out, as often seen with online surveys, ${ }^{35} 36$ an initial recruitment of 340 participants is required. We anticipate enrolling those participants from 10 PICUs over a 12-month period. It is expected that recruitment numbers will vary across the sites and across the recruitment period, accounting for seasonal admissions involving healthy children being admitted for bronchiolitis and other respiratory and/or septic illness in the 
winter months. Recruitment targets will be discussed at each site set-up, with the allowance of over-recruiting in larger sites where possible.

\section{Qualitative study}

A realistic and pragmatic sample size of 15-20 parents/ caregivers will be interviewed at 3 and 6 months after PICU discharge, with the aim of increasing research knowledge in this unknown field. We recognise that we may not achieve data saturation with this sample size, as there are many different influences and variables surrounding the child's PICU admission, parents/caregivers' feeding experiences and survivorship journeys. However, this limitation will be acknowledged, investigated and discussed in the data analysis and future reporting of any study results, including the impact this may have on the study's credibility and generalisability.

\section{Sampling strategy \\ Quantitative study}

Initially, convenience sampling will be used to identify and recruit previously healthy children aged $\geq 37$ weeks' gestational age and $\leq 4$ years who have survived an admission to PICU and their parents/caregivers. During the recruitment period, monthly progress will be monitored by the lead researcher (KM) and a proportional quota sampling strategy will be used to recruit a sample representative to the UK PICU population in terms of age. Recruitment strategies will be employed against the population strata taken from annual UK PICU admission data ${ }^{4}$ (table 2). To increase our understanding of the experiences that both fathers and mothers have after their child has survived intensive care, we are encouraging both fathers and mothers to complete the parental questionnaires where possible.

\section{Qualitative study}

A purposeful sampling strategy will be used to interview a range of parents/caregivers based on reason for admission, age of child admitted to PICU and gender of parent (table 3). This will ensure that not just mothers, or parents/caregivers of planned surgery or parents/caregivers of babies are only interviewed, for example.

\begin{tabular}{llll}
\hline Table 2 & Proportional quota sampling strategy \\
\hline Strata (age) & $\begin{array}{l}\text { UK PICU } \\
\text { population }\end{array}$ & $\begin{array}{l}\text { Pro-rata } \\
\text { sample }\end{array}$ \\
\hline Less than 1 year & $45 \%$ & 153 & 217 \\
1 year old & $11 \%$ & 37 & 53 \\
2 years old & $6 \%$ & 20 & 28 \\
3 and 4 years old & $9 \%$ & 30 & 42 \\
\hline Total & $70 \%$ & $240(70 \%)$ & $340(100 \%)$ \\
\hline
\end{tabular}

PICU, paediatric intensive care unit.

\begin{tabular}{|c|c|}
\hline Inclusion criteria & Rationale \\
\hline $\begin{array}{l}\text { Parents/caregivers of } \\
\text { children enrolled into the } \\
\text { PIES Study }\end{array}$ & $\begin{array}{l}\text { To be able to compare } \\
\text { experiences with quantitative data } \\
\text { from the survey }\end{array}$ \\
\hline Mothers and fathers & $\begin{array}{l}\text { To obtain experiences of both } \\
\text { mothers and fathers }\end{array}$ \\
\hline $\begin{array}{l}\text { Emergency and planned } \\
\text { admission }\end{array}$ & $\begin{array}{l}\text { To obtain experiences of } \\
\text { parents/caregivers dealing with } \\
\text { both planned and emergency } \\
\text { admission as there are often } \\
\text { psychological sequelae } \\
\text { associated with emergency } \\
\text { versus planned admissions to } \\
\text { PICU }\end{array}$ \\
\hline $\begin{array}{l}\text { Age of child: } \\
\text { } \leq 6 \text { months (or pre- } \\
\text { weaned babies) } \\
>>6 \text { months }-1 \text { year } \\
>>1-2 \text { years } \\
>>2-4 \text { years }\end{array}$ & $\begin{array}{l}\text { To obtain differing experiences } \\
\text { of feeding during significant } \\
\text { developmental feeding milestones } \\
\text { for example weaning versus } \\
\text { autonomous child self-feeding } \\
\text { during preschool years }{ }^{53}\end{array}$ \\
\hline
\end{tabular}

PICU, paediatric intensive care unit; PIES Study, feeding and survivorship outcomes in previously healthy young Paediatric IntensivE care Survivors.

\section{Study measures}

Longitudinal follow-up survey

The outcome measures for the longitudinal follow-up survey have been selected based on their validity, reliability, use in previous paediatric populations and ease of use for participants. Pre-existing validated questionnaires will be used to measure feeding difficulty assessment, parental stress, parental feeding styles and child behaviour. To obtain longitudinal outcome data and potentially identify acute and/or chronic feeding difficulties, data from the questionnaires will be collected at four time points: at PICU discharge (retrospective data), 1, 3 and 6 months after PICU discharge. The outcome measures and time points are outlined in table 4 . The questionnaires have also been selected according to age of the child participant, in addition to tested psychometric properties.

\section{Feeding difficulty assessment measures}

- Infant Feeding Questionnaire, ${ }^{37}$ (7 items; up to 9-month-old babies).

- Behavioural Paediatric Feeding Assessment Scale, ${ }^{38}$ (35 items; 9 months old to 7 years).

\section{Parental stress measure}

- Parental Stress Scale, ${ }^{39}$ (18 items).

\section{Parental feeding style measures}

- Infant Feeding Questionnaire, ${ }^{40}$ (25 items; up to 2 years).

- Child Feeding Questionnaire, ${ }^{41}$ (28 items; from 2 years onwards). 
Table 4 Data collection measures and time points

\begin{tabular}{|c|c|c|c|c|}
\hline Time point & data) & discharge) & discharge) & discharge) \\
\hline \multicolumn{5}{|l|}{ Enrolment: } \\
\hline Eligibility screening (daily) & $x$ & & & \\
\hline Recruitment & $x$ & & & \\
\hline \multicolumn{5}{|l|}{ Assessments: } \\
\hline Demographic information & $x$ & & & \\
\hline \multicolumn{5}{|l|}{ Feeding difficulty assessment measures } \\
\hline $\begin{array}{l}\text { Infant Feeding Questionnaire or } \\
\text { Behavioural Paediatric Feeding Assessment Scale }\end{array}$ & $x$ & $x$ & $x$ & $x$ \\
\hline $\begin{array}{l}\text { Infant Feeding Questionnaire or } \\
\text { Child Feeding Questionnaire }\end{array}$ & $x$ & $x$ & $x$ & $x$ \\
\hline \multicolumn{5}{|l|}{ Child behaviour measures } \\
\hline $\begin{array}{l}\text { Infant Behaviour Questionnaire (very short version) or } \\
\text { Early Childhood Behaviour } \\
\text { Questionnaire }\end{array}$ & $x$ & $\times$ & $x$ & $x$ \\
\hline \multicolumn{5}{|l|}{ Qualitative interviews: } \\
\hline Invitation & $x$ & & & \\
\hline Interviews & & & $x$ & $x$ \\
\hline
\end{tabular}

Baseline $\quad 1$ month 3 months 6 months $\begin{array}{llll}\text { (retrospective } & \text { (after PICU } & \text { (after PICU } & \text { (after PICU } \\ \text { data) } & \text { discharge) } & \text { discharge) } & \text { discharge) }\end{array}$

Time point

nrolment:

Eligibility screening (daily)

Recruitment

Assessments:

Demographic information

Parental/caregivers' reports of feeding history (prior to PICU

admission)

Feeding difficulty assessment measures

Infant Feeding Questionnaire or

Behavioural Paediatric Feeding Assessment Scale

Parental stress measure

Parental feeding style measures

Infant Feeding Questionnaire or

Child Feeding Questionnaire

Child behaviour measures

Infant Behaviour Questionnaire (very short version) or

Early Childhood Behaviour

Questionnaire

PICU, paediatric intensive care unit.

\section{Child behaviour measures}

- Infant Behaviour Questionnaire-very short version, ${ }^{42}$ (36 items; up to 12 months).

- Child Behaviour Questionnaire-very short version, ${ }^{43}$ (35 items; from 1 year).

\section{Demographic information}

At each survey, parental factors, family variables and socioeconomic data will be collected to identify any relationship between family background and the development of feeding difficulties for young survivors of critical illness. This includes parental/caregiver:

- Ethnic origin.

- Age.

- Gender.

- Highest level of education.

- Living situation.

- Employment status.

- Siblings in household.

Routinely collected clinical PICU data

For all recruited patients, data already recorded during the child's PICU admission will be captured on a paper or electronic Case Report Form completed by the research nurse, a clinical team member delegated by the local principal investigator or by the chief investigator at a later date. The variables of interest have been identified as:

- Length of PICU stay (in hours).

- Length of intubation (in hours).

- Length of mechanical invasive ventilation (in hours).

- Number of (re)intubations.

- Type of ETT (oral or nasal).

- Length of non-invasive ventilation (in hours and mode).

- Inotrope requirement (yes/no).

- Mode of feeding during PICU admission (enteral, bolus or continuous, parental nutrition, oral diet, location of feeding tube).

- Time from extubation to commence oral feeding (in hours).

- Mode of feeding at PICU discharge.

- Documented evidence of gastric intolerance (vomiting, diarrhoea, abdominal distention).

\section{Qualitative study}

The main aim of the semistructured qualitative interviews is to develop an in-depth understanding of how parents/ caregivers of previously healthy young children ( $\leq 4$ years 
old) who survive critical illness construct, experience and make sense of their survivorship journey from PICU admission, specifically looking at feeding experiences and parental-child behaviours. Parents will be interviewed at approximately 3 and 6 months post-PICU discharge so that they can describe how and/or if their experiences are changing (or have changed) along the PICU survivorship journey.

\section{Study procedures \\ Quantitative study}

Over a 12-month period, each site will screen all children admitted to PICU and invite all eligible children and their parents/caregivers to participate in the study. Site investigators (or their designated nominee) who are part of the PICU clinical care team will determine eligibility. Parents/caregivers could be approached to take part in the study when the child is still in PICU, near to or at discharge, on the high dependency unit or hospital ward soon after being discharged from PICU. Once informed consent has been obtained, parents/caregivers will be asked to complete baseline questionnaires (paper or online options available). Parent/caregiver contact details will be obtained and securely recorded on a password-protected database to enable follow-up survey distribution at 1, 3 and 6 months. Follow-up survey data will be collected using either online or paper questionnaires as agreed by the parents/caregivers at recruitment. Two fortnightly reminders will be sent for the follow-up surveys as reminder letters, telephone calls, messages or email by the lead researcher (KM) as agreed with the participant at recruitment. As there is such a small time frame between 1-month and 3-month assessments, if no response is received following the 1-month survey, participants will still be approached at 3 months. If there is no response at this point however, they will not be approached again at 6 months.

\section{Qualitative study}

During recruitment into the multicentred survey, parents/caregivers will be invited to take part in the qualitative interviews. Those who consent to an interview will be approached by the lead researcher (KM) at the time in which reminders of the follow-up survey are sent (at 1, 3 and 6 months) either by reminder letters, telephone call, messages or email as agreed at recruitment. Semistructured open-ended questions will be used as the primary method of data collection to allow the parent/caregiver to describe their story, communicate their experiences, feelings and PICU survivorship journey. In response to patient and public involvement (PPI) feedback highlighting the lack of spare time that parents/caregivers of young children often face, telephone and internet (ie, Microsoft teams: Microsoft 365, UK) interviews will be conducted at a time convenient for the parent/caregiver which could include evenings and weekends.

\section{Data analysis}

All data obtained will be analysed. In circumstances where participants are deemed lost to follow-up, any data supplied will be analysed and used where appropriate, even if it can only be used to describe the cohort at baseline. A pragmatic approach to missing data will be used, whereby data will be analysed as much as possible. Data from non-responders will be used within the analysis to observe for non-response bias.

\section{Quantitative study data analysis}

Descriptive statistics will be used to present the demographic data information taken from the routinely collected clinical PICU data. All child and parent/caregiver outcome measures will be calculated, including means, SD, medians and IQRs for continuous variables and frequency counts and percentages for categorical data. Data will be examined for normality, outliers and for missing data. Statistical analysis will be completed using the IBM SPSS and statistical significance will be set at $\mathrm{p}<0.05$.

Analyses related to the study specific objectives include the following:

Objective 1: To characterise and measure the prevalence of feeding difficulties in previously healthy children ( $\leq 4$ years) who survive critical illness during the first 6 months after PICU discharge. From the feeding difficulty assessment measures, descriptive statistics (frequency counts and percentages) will be used to identify the numbers and types of feeding difficulties at each time point collected and for different age groups.

Objective 2: To identify clinical predictors for the development of feeding difficulties in previously healthy young children ( $\leq 4$ years) who survive critical illness. The information from the routinely collected clinical PICU data will be used to identify any clinical predictors for the development of feeding difficulties, such as length of intubation and time to commence oral feeding. Statistical analysis will involve multiple \pm linear regressions to see if we can predict feeding difficulty questionnaire scores from the clinical variables.

Objective 3: To identify parental/caregiver feeding styles for previously healthy young children ( $\leq 4$ years) who survive critical illness. Descriptive statistics will be initially performed to identify the frequency of participants in each parental feeding style, to identify the majority. This will then be repeated at each time point collected, to identify a change (or not) in parental feeding style across the 6 months from PICU discharge. If we have enough data, differences between mother feeding styles and father feeding styles will be calculated using MannWhitney U test (non-parametric) or t-test (parametric) as appropriate. The relationship between parental feeding style and feeding difficulty score will also be tested using the same statistical tests.

Objective 4: To measure parental stress in parents/ caregivers of previously healthy young children ( $\leq 4$ years) who survive critical illness. Using the scores from the 
parental stress scale, average parental stress scores for all participants will be calculated at all time points. Average parental stress score at each time point, for those parents of children with and without feeding difficulties, will also be presented to identify the trajectories of parental stress over time and between the two groups. Correlation and regression analysis will be used to investigate relationships between increasing feeding difficulty score and increasing parental stress score.

Objective 5: To identify behaviours of previously healthy young children ( $\leq 4$ years) who survive critical illness. Frequency of children in each temperament category from the Infant and Early Child Behaviour Questionnaires will be calculated and presented at each data collection time point, so observe for changes over time. The relationship between infant/child temperament and feeding difficulty score; and parental feeding style and parental stress score will be assessed using Mann-Whitney $\mathrm{U}$ test (non-parametric) or t-test (parametric) and regression models where appropriate.

\section{Qualitative study data analysis}

All interviews will be audio-recorded and transcribed verbatim. All data will be imported into a qualitative data analysis package (NVivo), which will assist in managing, sorting and coding the vast data set. Data analysis will be largely conducted by KM, with the other researchers (A-SED, LVM) verifying the findings for consistencies and discrepancies to maximise credibility and reliability. ${ }^{44}$ Data analysis will involve three stages: (1) narrative analysis, (2) thematic analysis and (3) integration and will look to answer study:

Objective 6: to develop an in-depth understanding of how parents/caregivers of previously healthy young children ( $\leq 4$ years old) who survive critical illness construct, experience and make sense of their survivorship journey from PICU admission, specifically looking at feeding experiences and parental-child relationships.

\section{Stage 1: narrative analysis}

The first stage of analysis will involve analysing the content of the data from each participant's interview using the Clandinin and Connelly's ${ }^{45}$ method of narrative inquiry. This framework uses three domains to structure the analysis: temporality, sociality and place ${ }^{46}$ The analysis focuses on the actual storylines that are told and emotions that are used to tell the story, the societal and cultural impact on the story and the influence of the place in which the experience occurs. ${ }^{45}$ An additional consideration of the actual words and language, both verbal and non-verbal, used throughout the narrative will also be used during the analysis. ${ }^{45}$

\section{Stage 2: thematic analysis}

The second stage of analysis will involve a thematic analysis approach, whereby repeated patterns across the stage 1 analysis will be identified, leading to the detection of codes and themes across the entire data set. ${ }^{47}$ This will enable meaning and patterns to emerge from the data.

\section{Stage 3: data integration}

The final step of the qualitative data analysis will involve integrating the narrative and thematic analysis. The individual stories will be retold in a coherent manner and then the key themes across the entire data set will be presented. This will provide a detailed description and understanding of the survivorship journey of parents/ caregivers of previously health children who survive critical illness.

\section{Data integration strategy of quantitative and qualitative data}

The quantitative data from the survey and the qualitative data from the interviews will be analysed concurrently as they are collected and then integrated to answer the overarching research questions and aims. The qualitative data will strengthen the survey findings by adding the human perspective, exploring behaviour, feelings and experiences of the parents/caregivers told by them. ${ }^{48}$ The information gained from the interviews will assist interpretation and analysis of the survey results, drawing conclusions to the clinical significance of the results with implications for clinical practice. ${ }^{49}$

\section{Patient and public involvement}

Guided by the National Institute for Health Research INVOLVE recommendations, ${ }^{50}$ involvement of families of children recently discharged from PICU was sought during the study design process. Six parents volunteered to provide guidance and advice during an organised coffee morning. Collectively, the importance of the study was recognised, and recommendations made to the recruitment process and data collection methods. Feedback included using an online questionnaire for ease of use and to increase follow-up completion. The survey questions were also piloted by parents, assessing the clarity of the questionnaires and their instructions and to consider the burden of completing all four questionnaires. Offering home, telephone and internet interviews was also suggested for the interviews.

\section{Ethics and dissemination}

\section{Informed consent}

Parents/caregivers will be approached to take part in the PIES Study once the child meets the inclusion/exclusion criteria. After being given an ethically approved Participant Information Sheet (PIS), parents/caregivers will be given at least 48 hours to consider participation, unless they are happy to give informed consent before this time. It is anticipated that the children eligible for the study will be too young and/or too ill to participate directly in the consent process. Each parent/caregiver will complete a contact form that will record the information needed for the follow-up survey distribution (eg, mail addresses, telephone numbers) and informed consent will be obtained to allow the sharing of this personal data. 


\section{Researching sensitive and emotive topics}

It is recognised that parents/caregivers of previously healthy young children who have survived critical illness may have psychological sequelae (ie, post-traumatic stress disorder) following their child's admission to PICU. ${ }^{51}$ Although not specifically asking about their PICU experience, completing the survey and taking part in the interviews may raise potentially distressing issues around difficult feeding and/or mealtime behaviours following the PICU admission. Initial instances of distress will be dealt by the researcher and supported by the PICU psychology team at the researchers' host institution. The researcher will also signpost the participants to the Patient Advice and Liaison Services, clinical psychology team based at Southampton Children's Hospital and other local healthcare teams.

\section{Burden}

The survey is compiled of four separate pre-existing validated questionnaires, asked at four separate time points during the enrolment and follow-up (at recruitment, at 1, 3 and 6 months after PICU discharge). The questionnaires include Likert scales, yes/no answers and dropdown options. The survey questions and instructions have been piloted by parents of young children, assessing the clarity of the questionnaires, the instructions and consideration of the time and mental burden in completing all four questionnaires. Average time for survey completion was $15 \mathrm{~min}$, with follow-up surveys thought to be quicker. We endeavour to reduce this burden by having the option of an online electronic survey available to parents and by adding the feature where you can 'save and go back to later' option within the survey. The PIS will clearly state that there will be no financial gain from taking part in the study. Conversely, some participants might find taking part in the study beneficial because they will have the time and space to think about issues which are important to them.

\section{Ethical review}

The Yorkshire and The Humber-South Yorkshire Research Ethics Committee has reviewed the study protocol and provided favourable opinion (Ref: 20/ $\mathrm{YH} / 0160)$. The Health Research Authority has also approved the protocol (IRAS: 279171). This study has been extensively peer reviewed through the University of Southampton and forms the PhD study of the first author.

\section{Methods of dissemination}

This paper is part of the dissemination plan of the PIES Study, by presenting the project background, providing a detailed description of methods and procedures used to collect and analyse the data. Other dissemination plans involve local, national and international audiences including academics, healthcare professionals, healthcare commissioners, charities and the public. Dissemination will include written and oral feedback to the PPI group, local PICU charity and each recruitment centre. Presentations to local and national research and clinical teams will take place, including research meetings and conferences. The findings from this study will contribute to addressing the significant gaps in the literature by investigating the prevalence of and predictors for feeding difficulties experienced by previously healthy young children who survive critical illness and explore the effect on parental feeding experiences, behaviours and stress. It is anticipated that the expected outputs of this proposed project will be in terms of high-quality, peer-reviewed scientific publications and conference presentations. During the informed consent process, parents/caregivers will be asked if they would like a lay summary of any study findings sent to them at the end of the study.

\section{Author affiliations}

${ }^{1}$ Paediatric Intensive Care Unit, Southampton Children's Hospital, Southampton, UK ${ }^{2}$ School of Health Sciences, University of Southampton, Southampton, UK ${ }^{3} \mathrm{NIHR}$ Biomedical Research Centre, University Hospital Southampton NHS Foundation Trust, Southampton, UK

${ }^{4}$ Department of Dietetics and Speech and Language Therapy, University Hospital Southampton NHS Foundation Trust, Southampton, UK

\section{Twitter Kathryn Morton @Katystearn1Katy}

Acknowledgements The PIES Study protocol was developed by KM, LVM and A-SED. The corresponding author would like to acknowledge LVM and A-SED in their supervisory support during KM's PhD in which the PIES Study has been developed. KM would like to acknowledge the University of Southampton and University Hospital Southampton NHS Foundation Trust in supporting and funding her PhD through the Wessex Clinical Doctoral Research Fellowship scheme, and the clinical team at Southampton Children's Hospital PICU in allowing KM the clinical backfill time to undertake her PhD. Furthermore, KM would like to acknowledge Kevin Wheeler (Clinical Informatics Research Unit, Southampton) for his support and patience in developing the PIES Study data capture and management through the ALEA database/eCRF.

Contributors Authors made the following contribution to the manuscript: KM formulated the original research idea, conducted the literature searching and is the chief investigator for the study. KM drafted the manuscript from the ethically approved protocol (which was originally supported by LVM and A-SED). LVM, A-SED and KM reviewed and revised the manuscript for important intellectual content. All authors provided final approval of the version to be submitted.

Funding This report describes independent research arising from a personal Clinical Doctoral Research Fellowship for Kathryn Morton, supported jointly by the University of Southampton and University Hospital Southampton NHS Foundation Trust, England.

Competing interests None declared.

Patient and public involvement Patients and/or the public were involved in the design, or conduct, or reporting, or dissemination plans of this research. Refer to the Methods section for further details.

Patient consent for publication Not required.

Provenance and peer review Not commissioned; externally peer reviewed.

Open access This is an open access article distributed in accordance with the Creative Commons Attribution Non Commercial (CC BY-NC 4.0) license, which permits others to distribute, remix, adapt, build upon this work noncommercially, and license their derivative works on different terms, provided the original work is properly cited, appropriate credit is given, any changes made indicated, and the use is non-commercial. See: http://creativecommons.org/ licenses/by-nc/4.0/.

ORCID iD

Kathryn Morton http://orcid.org/0000-0003-0970-0081 


\section{REFERENCES}

1 Kanthimathinathan HK, Plunkett A, Scholefield BR, et al. Trends in long-stay admissions to a UK paediatric intensive care unit. Arch Dis Child 2020;105:558-62.

2 Davis P, Stutchfield C, Evans TA, et al. Increasing admissions to paediatric intensive care units in England and Wales: more than just rising a birth rate. Arch Dis Child 2018;103:341-5.

3 Colville G, Pierce C. Patterns of post-traumatic stress symptoms in families after paediatric intensive care. Intensive Care Med 2012;38:1523-31.

4 (PICANET) PICAN. Annual report 2017, 2017. Available: http://www. picanet.org.uk/Audit/Annual-Reporting/PICANet_2017_Annual_ Report_Summary_v1.0_FINAL.pdf

5 Ruhl AP, Lord RK, Panek JA, et al. Health care resource use and costs of two-year survivors of acute lung injury. An observational cohort study. Ann Am Thorac Soc 2015;12:392-401.

6 Manning JC, Hemingway P, Redsell SA. Survived so what? identifying priorities for research with children and families postpaediatric intensive care unit. Nurs Crit Care 2018;23:68-74.

7 Rennick JE, Johnston CC, Dougherty G, et al. Children's psychological responses after critical illness and exposure to invasive technology. J Dev Behav Pediatr 2002;23:133-44.

8 Leder SB. Incidence and type of aspiration in acute care patients requiring mechanical ventilation via a new tracheotomy. Chest 2002;122:1721-6.

9 Tolep K, Getch CL, Criner GJ. Swallowing dysfunction in patients receiving prolonged mechanical ventilation. Chest 1996;109:167-72

10 Skoretz SA, Flowers HL, Martino R. The incidence of dysphagia following endotracheal intubation: a systematic review. Chest 2010;137:665-73.

11 Macht M, Wimbish T, Clark BJ, et al. Postextubation dysphagia is persistent and associated with poor outcomes in survivors of critical illness. Crit Care 2011;15:R231.

12 Merriweather JL, Salisbury LG, Walsh TS, et al. Nutritional care after critical illness: a qualitative study of patients' experiences. J Hum Nutr Diet 2016;29:127-36.

13 Merriweather J, Smith P, Walsh T. Nutritional rehabilitation after ICU does it happen: a qualitative interview and observational study. J Clin Nurs 2014;23:654-62.

14 Briassoulis GC, Zavras NJ, Hatzis MD TD. Effectiveness and safety of a protocol for promotion of early intragastric feeding in critically ill children. Pediatr Crit Care Med 2001;2:113-21.

15 Meyer R, Harrison S, Sargent S, et al. The impact of enteral feeding protocols on nutritional support in critically ill children. J Hum Nutr Diet 2009;22:428-36.

16 Mehta NM, Skillman HE, Irving SY, et al. Guidelines for the provision and assessment of nutrition support therapy in the pediatric critically ill patient: Society of critical care medicine and American Society for parenteral and enteral nutrition. Pediatr Crit Care Med 2017;18:675-715.

17 Tregay J, Wray J, Crowe S, et al. Going home after infant cardiac surgery: a UK qualitative study. Arch Dis Child 2016;101:320-5.

18 Powell-Tuck J. Nutritional interventions in critical illness. Proc Nutr Soc 2007;66:16-24.

19 Senez C, Guys JM, Mancini J, et al. Weaning children from tube to oral feeding. Childs Nerv Syst 1996;12:590-4.

20 Dello Strologo L, Principato F, Sinibaldi D, et al. Feeding dysfunction in infants with severe chronic renal failure after long-term nasogastric tube feeding. Pediatr Nephrol 1997;11:84-6.

21 Thoyre SM. Developmental transition from gavage to oral feeding in the preterm infant. Annu Rev Nurs Res 2003;21:61-92.

22 Savage JS, Fisher JO, Birch LL. Parental influence on eating behavior: conception to adolescence. J Law Med Ethics 2007;35:22-34.

23 Lutz KF. Feeding problems of NICU and PICU graduates: perceptions of parents and providers. Newborn Infant Nurs Rev 2012;12:207-13.

24 Long KA, Marsland AL. Family adjustment to childhood cancer: a systematic review. Clin Child Fam Psychol Rev 2011;14:57-88.

25 Morton K, Marino LV, Pappachan JV, et al. Feeding difficulties in young paediatric intensive care survivors: a scoping review. Clin Nutr ESPEN 2019;30:1-9.

26 Plano-Clark C. Designing and conducting mixed methods research. 2nd ed. Thousand Oaks, California: SAGE publishing, 2011.
27 Story M, Holt K, Sofka D. Bright futures in clinical practice. Nutrition, Rockville, MD: National Center for Education in Maternal and Child Health, 2002.

28 Wickenden M. The development and disruption of feeding skills in babies and young children. In: Southall A, Clarrissa M, eds. Feeding problems in children: a practical guide (2nd edition). Oxford: Radcliffe Publishing, 2011.

29 FBH SM. Family routines and rituals: a context for development in the lives of young children. Infants \& Young Children 2007;20:284-99.

30 Hawdon JM, Beauregard N, Slattery J, et al. Identification of neonates at risk of developing feeding problems in infancy. Dev Med Child Neurol 2000;42:235-9.

31 Einarson KD, Arthur HM. Predictors of oral feeding difficulty in cardiac surgical infants. Pediatr Nurs 2003;29:315-9.

32 Kogon BE, Ramaswamy V, Todd K, et al. Feeding difficulty in newborns following congenital heart surgery. Congenit Heart Dis 2007;2:332-7.

33 Jadcherla SR, Vijayapal AS, Leuthner S. Feeding abilities in neonates with congenital heart disease: a retrospective study. J Perinatol 2009;29:112-8.

34 Indramohan G, Pedigo TP, Rostoker N, et al. Identification of risk factors for poor feeding in infants with congenital heart disease and a novel approach to improve oral feeding. J Pediatr Nurs 2017;35:149-54

35 Smeeth $\mathrm{L}$, Fletcher AE. Improving the response rates to questionnaires. BMJ 2002;324:1168-9.

36 Pulham RA, Wray J, Feinstein Y, et al. Feasibility and acceptability of methods to collect follow-up information from parents 12 months after their child's emergency admission to pediatric intensive care. Pediatr Crit Care Med 2019;20:e199-207.

37 Wright CM, Parkinson KN, Drewett RF. How does maternal and child feeding behavior relate to weight gain and failure to thrive? data from a prospective birth cohort. Pediatrics 2006;117:1262-9.

38 Crist W, MCDONNELL P, Beck M, et al. Behavior at Mealtimes and the young child with cystic fibrosis. J Dev Behav Pediatr 1994;15:157-61.

39 Berry JO, Jones WH. The parental stress scale: initial psychometric evidence. J Soc Pers Relat 1995;12:463-72.

40 Baughcum AE, Powers SW, Johnson SB, et al. Maternal feeding practices and beliefs and their relationships to overweight in early childhood. J Dev Behav Pediatr 2001;22:391-408.

41 Birch LL, Fisher JO, Grimm-Thomas K, et al. Confirmatory factor analysis of the child feeding questionnaire: a measure of parental attitudes, beliefs and practices about child feeding and obesity proneness. Appetite 2001;36:201-10.

42 Putnam SP, Helbig AL, Gartstein MA, et al. Development and assessment of short and very short forms of the infant behavior questionnaire-revised. J Pers Assess 2014;96:445-58.

43 Putnam SP, Rothbart MK. Development of short and very short forms of the children's behavior questionnaire. J Pers Assess 2006;87:102-12.

44 Mays NP C. Qualitative research in health care. London: BMJ Publishing Group, 1996.

45 Clandinin DJ CF, Inquiry N. Experience and story in qualitative research. San Francisco CA: Jossey-Bass, 2000.

46 Haydon G, Browne G, van der Riet P. Narrative inquiry as a research methodology exploring person centred care in nursing. Collegian 2018;25:125-9.

47 Virginia B, Clarke V. Using thematic analysis in psychology. Qual Res Psychol 2006;3:77-101.

48 Holloway laW S. Qualitative research in nursing and healthcare. 3rd edition. Oxford: Wiley-Blackwell, 2010.

49 Polit DaB CT. Essentials of nursing Reseach: appraising evidence for nursing practice. 9th edition. Philadephia: Wolters Kluwer, 2017.

50 INVOLVE. Briefing notes for researchers: involving the public in NHS, public health and social care research. Eastleigh: INVOLVE, 2012.

51 Shudy M, de Almeida ML, Ly S, et al. Impact of pediatric critical illness and injury on families: a systematic literature review. Pediatrics 2006;118 Suppl 3:S203-18.

52 Health Do. Research governance framework for health and social care. London: $\mathrm{DoH}, 2005$.

53 Wickenden $\mathrm{M}$. The development and disruption of feeding skills in babies and young children. In: Southall A, Clarrissa M, eds. Feeding problems in children; a practical guide. 2nd ed. Oxford: Radcliffe Publishings, 2011: 2-28. 